\section{Case Reports in Ophthalmology}

\title{
Corneal Neovascularization with Associated Lipid Keratopathy in a Patient with Obstructive Sleep Apnea-Hypopnea Syndrome Using a Continuous Positive Airway Pressure Machine
}

\author{
Konstantinos Oikonomakis ${ }^{\mathrm{a}}$ Myrsini Petrelli $^{\mathrm{a}} \quad$ Konstantinos Andreanos $^{\mathrm{a}}$ \\ Andreas Mouchtouris ${ }^{a}$ Petros Petrou ${ }^{a}$ Ilias Georgalas ${ }^{a}$ \\ Dimitrios Papaconstantinou $^{a} \quad G^{2}$ orge Kymionis ${ }^{a, b}$ \\ ${ }^{a}$ First Department of Ophthalmology, University of Athens, General Hospital of Athens

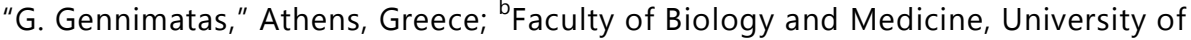 \\ Lausanne, Fondation Asile des Aveugles, Hôpital Ophtalmique Jules-Gonin \\ Lausanne, Switzerland
}

\section{Keywords}

Corneal neovascularization - Continuous positive airway pressure mask · Obstructive sleep apnea-hypopnea syndrome $\cdot$ Secondary lipid keratopathy

\begin{abstract}
Objective: To report a case of corneal neovascularization with secondary lipid keratopathy in a patient treated with continuous positive airway pressure (CPAP) for obstructive sleep apnea-hypopnea syndrome (OSAHS). Case Report: A 49-year-old male had been diagnosed with obstructive sleep apnea syndrome 10 years ago and has been treated with the application of a CPAP machine during night sleep ever since. For the past year, the patient had been complaining for ocular irritation and excessive tearing of the left eye on awakening. Slit-lamp biomicroscopy revealed the presence of neovascularization and lipid exudation in the inferior third of the cornea of the left eye. Ocular patching during night sleep resulted in recession of
\end{abstract}


the reported symptoms and shrinkage of the neovascularization, while the area of lipid exudation ceased to enlarge. Conclusion: To the best of our knowledge, this is the first report of corneal neovascularization in a patient using a CPAP machine for OSAHS.

\author{
(C) 2017 The Author(s) \\ Published by S. Karger AG, Basel
}

\title{
Introduction
}

Obstructive sleep apnea-hypopnea syndrome (OSAHS) has been associated with numerous ocular findings, including floppy eyelid syndrome (prevalence ranging from 2.3 to $53 \%$ in the literature), open-angle or normal-tension glaucoma (prevalence rate 3.4$16.67 \%$ ), senile cataract (10\%), nonarteritic ischemic optic neuropathy (up to $13.33 \%$ ), papilledema (up to $10 \%$ ), recurrent corneal erosion, keratoconus $(2.2 \%)$, central serous chorioretinopathy, and retinal vein occlusion. Furthermore, the majority of patients $(60 \%)$ suffering from OSAHS present some form of dry eye manifestations such as conjunctival hyperemia, lid parallel conjunctival folds, or conjunctivochalasis [1, 2].

In this case report, we describe - to our knowledge for the first time in the literature the emergence of corneal vascularization and lipid exudation in a patient treated with the application of a nasal continuous positive airway pressure (CPAP) mask for OSAHS.

\section{Case Report}

A 49-year-old patient sought medical consultation due to excessive tearing of the left eye noticed on awakening, especially after the nightly application of his CPAP machine. The morning symptoms appeared to be less prominent when he had not been using the CPAP machine overnight. In addition, the patient has noticed the presence of a white scar in his left eye since last year. He however reported no previous ophthalmic evaluation. In terms of the patient's medical history, he suffers from obstructive sleep apnea. The syndrome was diagnosed 10 years ago and the patient has been treated with the nightly application of a CPAP machine while sleeping in a supine position ever since. He reported replacement of the initial CPAP mask 18 months ago due to malfunction.

Ophthalmic examination of the left eye revealed an uncorrected distance visual acuity of $20 / 32$ and a corrected distance visual acuity of 20/20. Floppy eyelid syndrome and lagophthalmos were observed in both eyes, while the Bell phenomenon was present but decreased. Slit-lamp biomicroscopy showed no pathology in the right eye, whereas in the left eye, deep stromal vascularization with concomitant lipid deposition was noticed on the inferior third of the cornea (Fig. 1).

Due to the association between the morning appearance of the symptoms and the prior nightly use of the CPAP machine, we recommended our patient to use an eye pad to cover his left eye while asleep. A lubricating ointment at bedtime prior to eye covering was administered. One month later, the patient reported the disappearance of morning symptoms. Slitlamp biomicroscopy revealed diminution of the abnormal corneal vessels along with cessation of enlargement of the area of lipid exudate (Fig. 2). 


\section{Discussion}

CPAP is the treatment of choice for moderate-to-severe obstructive sleep apnea in adults. CPAP maintains the airway patency by delivering an intraluminal pressure that is positive with reference to the atmospheric pressure [3].

The effects of CPAP treatment on the eye have been randomly described in the literature. Kiekens et al. [4] reported that CPAP use results in an increase in intraocular pressure. Behbehani et al. [5] described the occurrence of ischemic optic neuropathy in 3 patients on CPAP treatment. Choroidal effusion [6] and periorbital edema [7] have also been associated with CPAP therapy. Regarding the effects of CPAP treatment on the ocular surface, the literature contains only a few case reports of ocular surface problems related to CPAP therapy. Harrison et al. [8] reported three obstructive sleep apnea cases on CPAP treatment that developed ocular surface complications. The first patient, a woman with keratoconus who used gas-permeable contact lenses, experienced vascularized limbal keratitis after the commencement of CPAP therapy. The second patient presented with recurrent corneal bacterial ulcers, while the third patient, who was diagnosed with pellucid marginal degeneration and used gas-permeable contact lenses, experienced contact lens intolerance and recurrent bacterial keratitis after the initiation of CPAP therapy. Fayers et al. [9] described reactivation of recurrent corneal erosion syndrome after CPAP therapy. Lastly, Hayirci et al. [10] reported increased incidence of squamous metaplasia in the conjunctiva of patients on CPAP treatment.

In terms of the effects of CPAP treatment on the tear film, the literature contains conflicting results. Hayirci et al. [10] found lower tear break-up time (TBUT) values following CPAP compared to baseline. This decrease in TBUT values was attributed to the irritative properties of the CPAP mask. On the contrary, both Kadyan et al. [2] and Acar et al. [3] reported increased TBUT values and a more stable tear film in patients with OSAHS after the application of CPAP treatment. The increased TBUT values were attributed to the necessity of sleeping in the supine position during PAP therapy as well as to the improvement of the clinical situation of floppy eyelid syndrome that led to ocular surface healing.

In our case, the patient reported air leakage from the nasal CPAP mask towards the left globe and the corresponding periocular area, a finding we were able to confirm when we examined our patient while lying and wearing the CPAP mask (Fig. 3). Alternatively, air may have found its way to the globe up through the nasolacrimal duct [1]. The aforementioned air leakage, in combination with the presence of lagophthalmos and diminished Bell phenomenon, may have resulted in chronic mechanical irritation of the left cornea. Our hypothesis is that the direct surface trauma induced chronic inflammation, leading to inflammatory deep stromal neovascularization and subsequent lipid deposition in the corneal stroma. Our recommendation to the patient was to use protective eyewear and to choose an appropriate type and size of CPAP mask.

In conclusion, all patients using a CPAP mask should be carefully evaluated for the development of abnormal vessels within the cornea that can be the result of chronic airinduced surface trauma by inappropriate application of the CPAP mask.

\section{Statement of Ethics}

Written informed consent was obtained from the patient for publication of this case report and accompanying images. 


\section{Case Reports in Ophthalmology}

Disclosure Statement

The authors have no conflict of interest to disclose.

\section{References}

1 Elsayed EM, Massoud TM: Effect of sleep related breathing disorders on ocular function. Egypt J Chest Dis Tuberc 2014;63:663-669.

2 Kadyan A, Asghar J, Dowson L, Sandramouli S: Ocular findings in sleep apnoea patients using continuous positive airway pressure. Eye (Lond) 2010;24:843-850.

-3 Acar M, Firat H, Yuceege M, Ardic S: Long-term effects of PAP on ocular surface in obstructive sleep apnea syndrome. Can J Ophthalmol 2014;49:217-221.

-4 Kiekens S, De Groot V, Coeckelbergh T, Tassignon MJ, van de Heyning P, De Backer W, Verbraecken J: Continuous positive airway pressure therapy is associated with an increase in intraocular pressure in obstructive sleep apnoea. Invest Ophthalmol Vis Sci 2008;49:934-940.

-5 Behbehani R, Mathews MK, Sergott RC, Savino PJ: Non-arteritic anterior ischemic optic neuropathy in patients with sleep apnea while being treated with continuous positive airway pressure. Am J Ophthalmol 2005;139:518-521.

-6 Simaroj P, Preechawat P: Choroidal effusion following continuous positive airway pressure treatment. J Med Assoc Thai 2009;92:987-989.

7 Ely JR, Khorfan F: Unilateral periorbital swelling with nasal CPAP therapy. J Clin Sleep Med 2006;2: 330-331.

8 Harrison W, Pence N, Kovacich S: Anterior segment complications secondary to continuous positive airway pressure machine treatment in patients with obstructive sleep apnea. Optometry 2007;78: 352-355.

-9 Fayers T, Simcock DE, Wilkins MR: Reactivation of recurrent corneal erosion syndrome by continuous positive pressure ventilation. Cornea 2007;26:1292.

10 Hayirci E, Yagci A, Palamar M, Basoglou OK, Veral A: The effect of continuous positive airway pressure treatment for obstructive sleep apnea syndrome on the ocular surface. Cornea 2012;31:604-608.
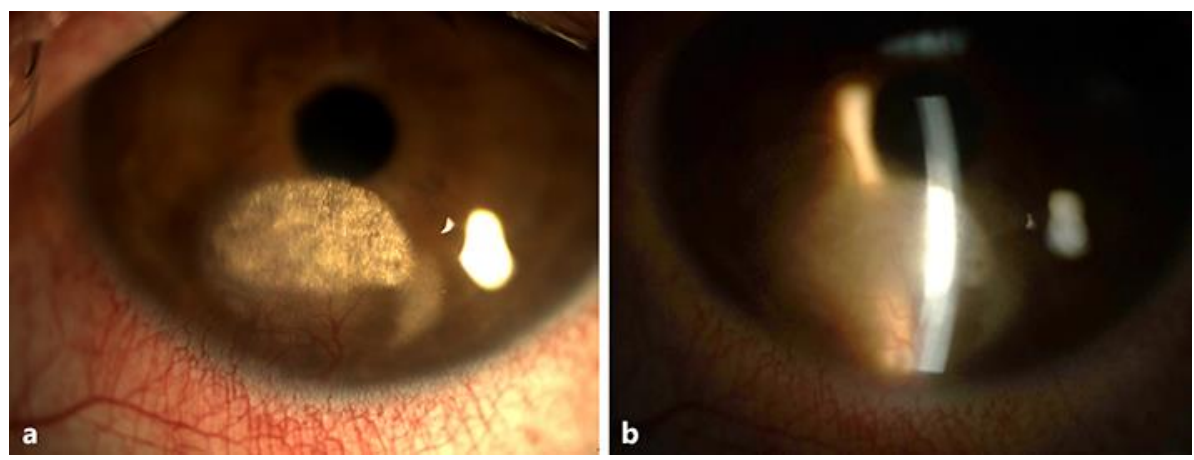

Fig. 1. Deep corneal stromal vascularization and lipid deposition. a Diffuse illumination. b Direct illumination. 
Case Reports in
Ophthalmology

Case Rep Ophthalmol 2017;8:416-420 DOI: $10.1159 / 000478925$

C 2017 The Author(s). Published by S. Karger AG, Basel www.karger.com/cop

Oikonomakis et al.: Corneal Neovascularization with Associated Lipid Keratopathy in a Patient with OSAHS Using a Continuous Positive Airway Pressure Machine

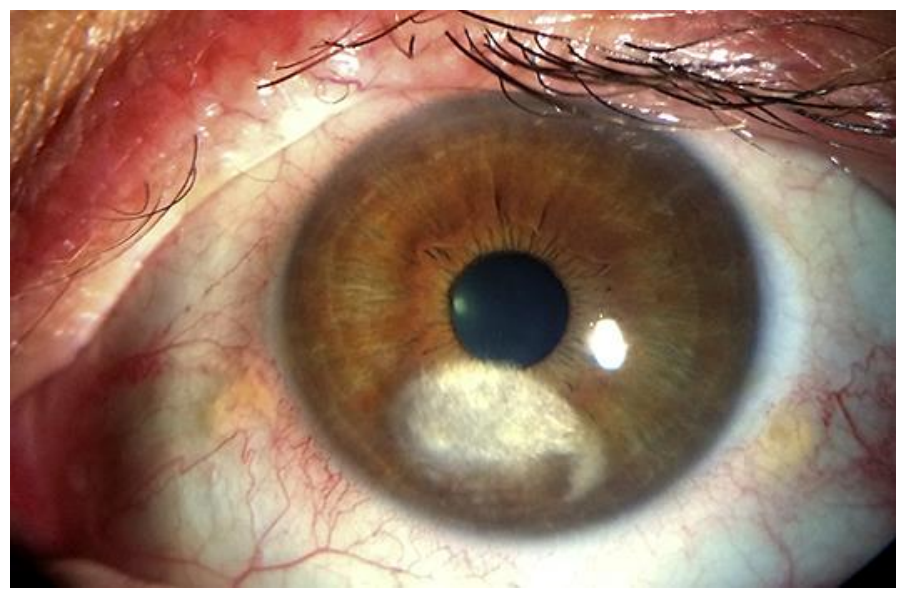

Fig. 2. Diminution of abnormal corneal vessels following bedtime eye covering.

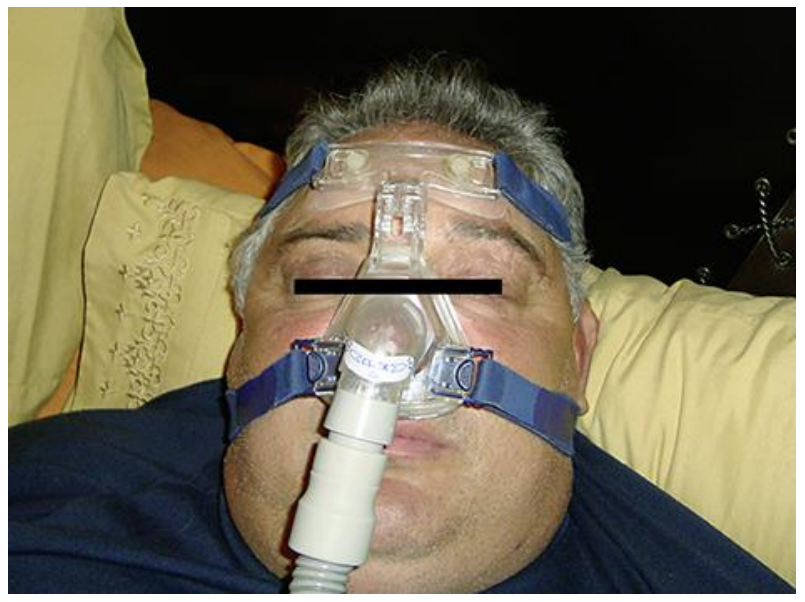

Fig. 3. Loose mask application leading to air leakage towards the left hemiface. 